\title{
HIGH PULSE POWER MODULATOR UPGRADE FOR A S-BAND TRANSMITTER
}

\author{
J. DeCobert, B. Binns, R. Campbell, A. Hawkins, D. Wang, A. Zolfaghari \\ MIT-Bates Linear Accelerator Center \\ Middleton, MA 01949, USA
}

\section{INTRODUCTION}

This paper describes transmitter systems upgrade plans for the Bates Linear Accelerator. There are six modulator systems. They are dual output S-band systems with a peak operating power of $4.5 \mathrm{MW}$ and $100 \mathrm{KW}$ average. They are floating-deck hard-tube modulators operating a parallel pair of Litton L-5097 switchtubes in series with a Varian VA-938 klystron.

\section{UPGRADE PHILOSOPHY}

The major goal of the modulator deck upgrade is to increase system reliability. System downtime needs to be significantly reduced to improve the overall efficiency of beam delivery. This will be accomplished by identifying the failure prone components and circuitry, and applying modern circuit design methodologies. This will also permit the elimination of outdated circuit components which are becoming increasingly harder to find. In addition, time to repair must be significantly reduced to minimize downtime. This will be accomplished in two ways. First, by optimizing ease of repair. Presently, transmitter subsystems generally use bolt-in-place components, which are difficult and time consuming to replace. These will be replaced by chassis with quick disconnects and plug-in cards. Second, by improving our capability to analyze quickly fault conditions. This will be accomplished by adding more diagnostics, and by using technologies that were not available for the original design [Figs. 3 and 4].

\section{PRESENT OPERATION}

The six transmitters were designed in the late 1960's and were installed in the early 1970 's. The basic transmitter consists of a $176 \mathrm{KV}$ power supply which has its positive terminal connected to ground. The power supply negative terminal B- is connected directly to the cathode of four switchtubes. Each transmitter contains two klystrons. Each klystron's collector is connected to $\mathrm{B}+$ and its cathode is connected to the anodes of two switchtubes. The modulating anodes of all four switch tubes receive their drive signals from a common switchtube driver circuit. The switch tube drive circuit, regulator, and other modulator circuits are mounted on a deck which floats at high voltage. This design has proven reliable and we do not wish to change it. The problem is trying to maintain beam delivery with
30 year old equipment, equipped with a 30 year old diagnostics system, interlock system, and monitoring system.

The present operation of the modulator deck is as follows [Fig. 3]. A pulsed signal is sent to the bootstrap chassis via a pulsed thyratron to a photomultiplier (PM) tube on the floating deck. The PM tube signal is amplified and sent to the bootstrap chassis, which initiates the leading edge of the pulse. When the pulse voltage reaches a set voltage, the Regulator chassis begins operation to keep the flattop portion of the pulse to within $0.1 \%$. At the time of turn-off, a pulse is sent via a separate thyratron/PM link to the tailbiter chassis, which initiate the falling edge and returns the modulator to its original state [Fig. 1].

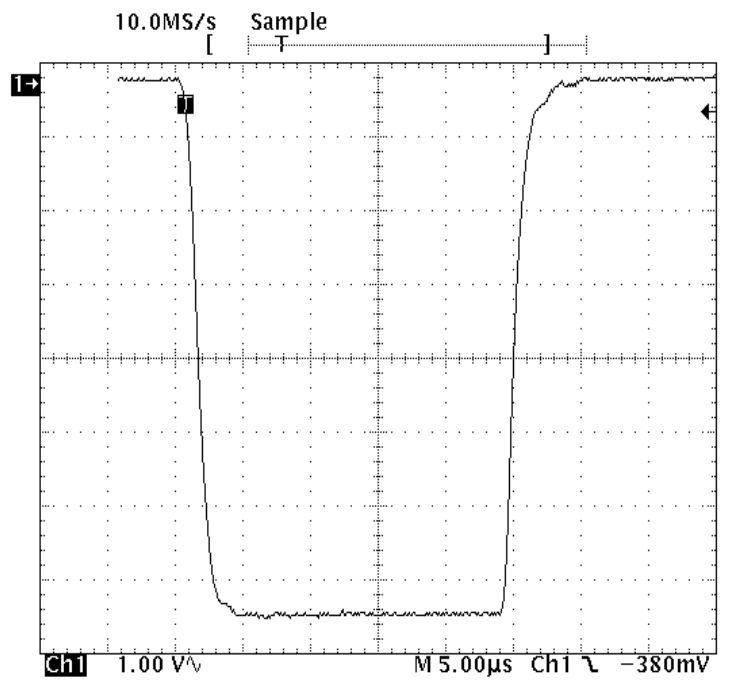

Fig. 1. Klystron cathode current pulse, 70 A peak.

\section{START AND STOP SIGNALS}

As mentioned earlier, the start and stop signals are transmitted to the floating deck electronics via a pulsed thyratron. The light output of the thyratron is directed down a Lucite rod to a photomultiplier tube on the floating electronics. This hardware will be replaced with fiber optics which will eliminate the need for high voltages for the thyratron and photomultiplier tubes. It will also eliminate problems in the alignment of the Lucite rods between the tubes, and extraneous signals being received by the photomultpliers. 


\section{BOOTSTRAP AND TAILBITER}

The subsystems which are responsible for the switching of the floating deck, the bootstrap and tailbiter chassis, are two of the most failure prone of the modulator. To replace one failed unit requires a complete system shutdown. Typical turn around times of such an operation are in excess of $11 / 2$ hours.

By mounting the circuits on plug-in cards, the need for total system shutdown and removal of the modulator deck should be eliminated. A rapid replacement of the circuits by putting the system into standby mode, or by shutting off only a few of the power supplies, should be possible. This would reduce the turnaround time by at least $75 \%$. Card mounted circuits should allow us to bench test the operation of these circuits, obviating the need to install them into a working spare deck, as is done now.

An effort to include inherent circuit redundancy is being made. For the critical trigger circuits, we will include a standby parallel circuit that may be either manually or automatically switched. This will allow for effective repair of the system without the need of shutting the system down or spending considerable time in removing the faulty circuits. It may also be simple enough for the operating staff to operate, so that time is not spent in waiting for repair personnel to arrive.

\section{REGULATOR}

The shunt regulator subsystem of the modulator deck will also be updated. This subsystem is responsible for the exceptional pulse flatness of the modulator. It currently has a vacuum tube based error amplifier. This error amplifier is somewhat difficult to troubleshoot, and balance, requiring a day long service to adjust. When the error amplifier fails, its repair time is on the order of a least one day. In emergencies the entire modulator deck electronics package may be replaced. This subsystem could be greatly simplified by using present day high voltage solid state methods. This will eliminate the long tuning procedure, and allow for a rapid replacement of failed units. Such solid state methods are already in use in similar systems. [Ref. 1]

\section{INTERLOCKS}

The modulator deck interlock system will be updated. In the existing system, the presence of required voltages are sensed through incandescent light falling on a photoresister in series with a sealed electromechanical relay. This system allows for wide variations in the required voltages, and has become difficult to maintain as original manufacturers no longer make many of the system elements, such as the electromechanical relays. In addition, no information from the interlock chain is transmitted to the ground level control, other than a SUM indication. By using modern opto-isolators, fiber optics and solid state relays, positive interlock window control will be implemented. In addition, each interlock, or at least subsets, can be transmitted via fiber optics to the control system. This will allow operators to be provided with better information on the system status, as well as provide helpful information for troubleshooting and repair.

\section{SYSTEM DIAGNOSTICS AND MONITORING}

A major reason for the lack of diagnostic and monitoring information designed into the system was the difficulty of bringing signals floating at high voltage to ground. Today, transmitters are designed using fiber optic technology to isolate signals floating at high voltage from ground. Hewlett-Packard and others have developed low cost, very reliable fiber optic transmitters and receivers. Manufacturer test data indicates for 12 lots of 330 HFBR-2402 fiber optic receivers, tested for 2.370,000 hours at 85 degrees Celsius, resulted in one failure. [Ref. 2]

To better understand the operation and failure modes of the deck, new fiber optic technology will be used to transmit the modulator deck status, as well as critical voltage and current parameters. This will be implemented by using precision voltageto-frequency and RMS-to-DC converters on the floating electronics, and transmitting via fiber optic to ground referenced circuitry. In this manner, critical voltage and current levels can be monitored while the modulator is in operation. Analog fiber optics will be used to send pulsed signals from the floating electronics to be monitored from the ground level. This technique has been used successfully in other systems [Ref. 3]. By adding this capability, it should be possible to rapidly analyze faults in the system [Fig.2]

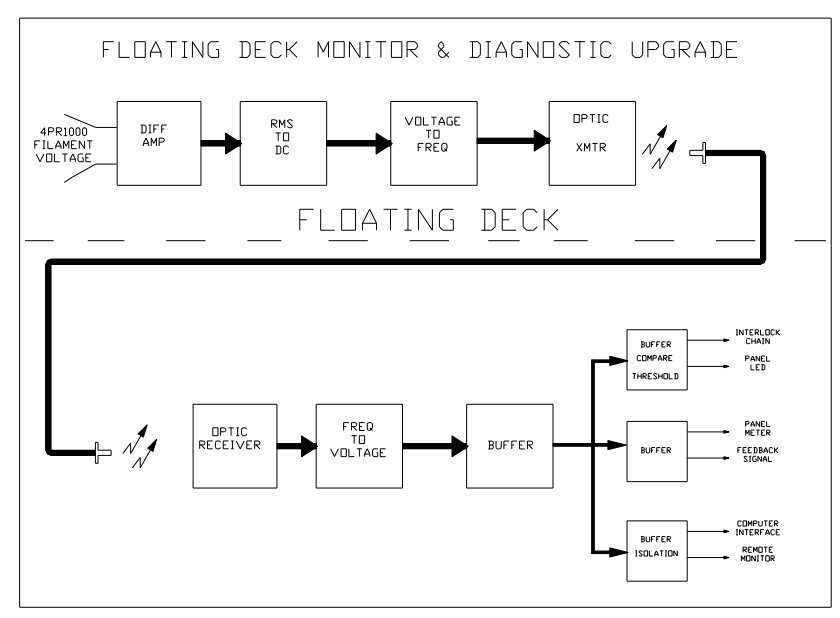

Fig. 2 Use of fiber optics in diagnostics and monitoring. 


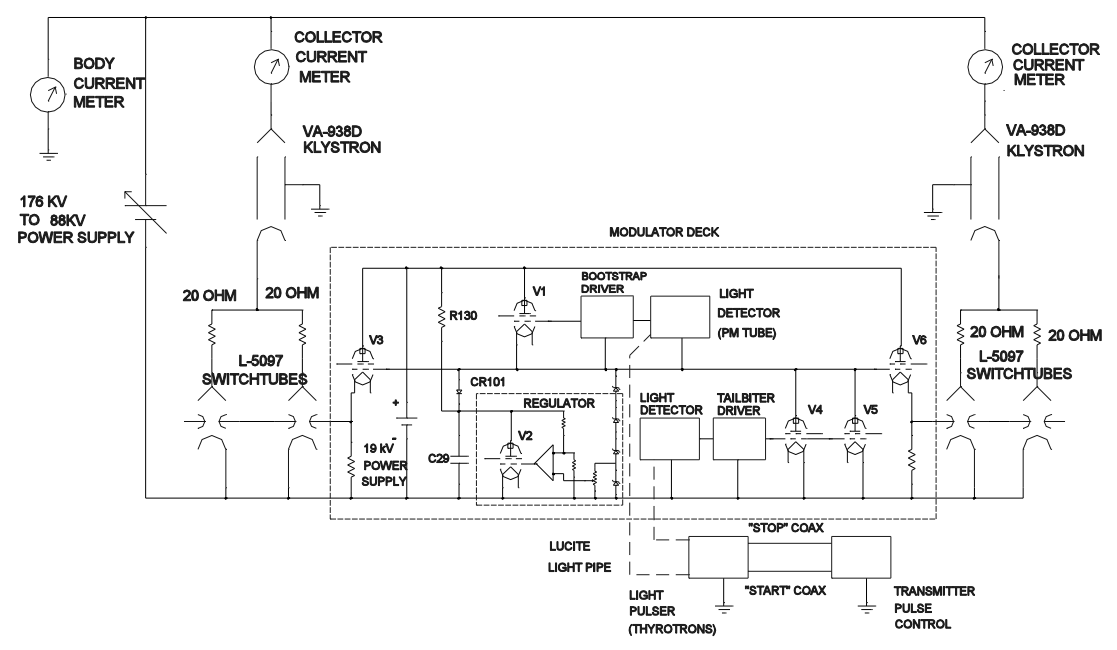

Simplified modulator schematic

Fig. 3

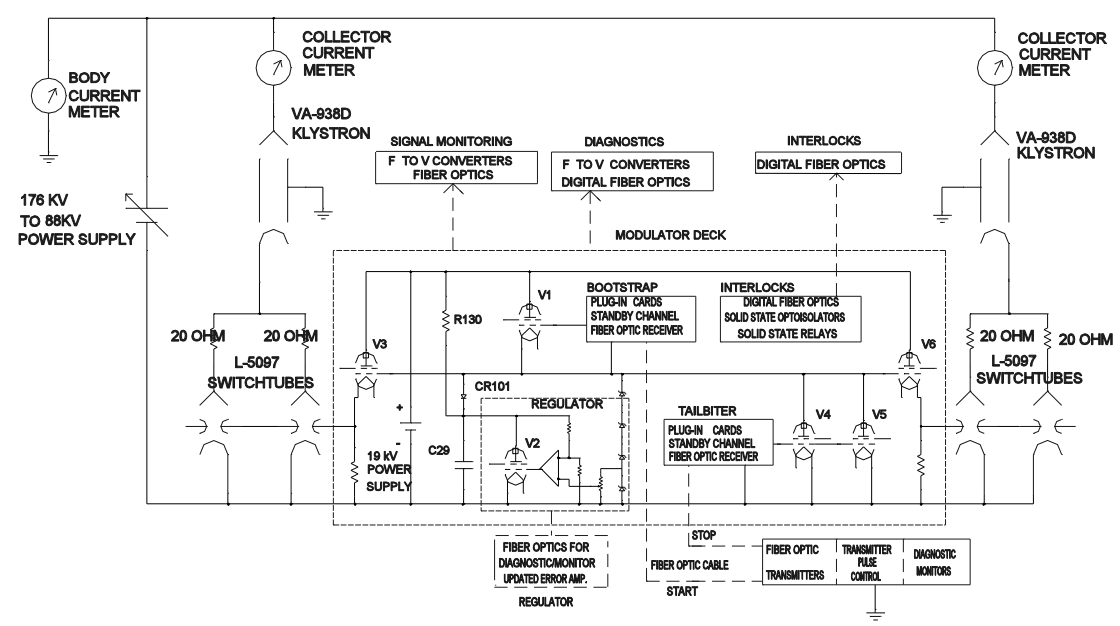

Simplified modulator schematic showing upgrades

Fig. 4

\section{REFERENCES:}

1. Bill North, GTE Government Systems, Bill Reass, Los Alamos National Laboratory, "Electrical Design and Operation of a Two-Klystron RF Station fro the Los Alamos National Laboratory's Neutral Particle Beam Experiment" 1992 IEEE Twentieth Power Modulator Symposium, Conference Record, P.15
2. Hewlett-Packard, "Fiber Optic Transmitter and Receiver Reliability Data" Bulletin Number 5954-2221 (8/89).

3. R. Litte and R. Limpaecher, Avco Research Laboratory, Inc. "Fiber Optic Transmission of High Speed Analog Data in High Voltage Pulsed Power Systems" 1986 IEEE Seventeenth Power Modulator Symposium, Conference Record, P.287 